\title{
CRIOTERAPIA EM MODELO DE COMPRESSÃO DO NERVO ISQUIÁTICO: ANÁLISE FUNCIONAL E MORFOLÓGICA
}

\author{
CRYOTHERAPYIN SCIATICNERVECOMPRESSIONMODEL:FUNCTIONAL ANDMORPHOLOGICAL ANALYSIS
}

CRIOTERAPIA EN MODELODE COMPRESIÓN DEL NERVIO CIÁTICO:ANÁLISIS FUNCIONAL YMORFOLÓGICO

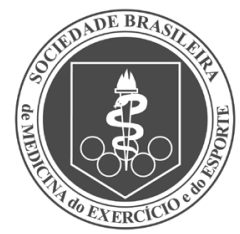

Artigo Original

Original ARticle Artículo Original
Jhenifer Karvat ${ }^{1}$

(Fisioterapeuta)

Lizyana Vieira?

(Fisioterapeuta)

Camila Mayumi Martin Kakihata

(Fisioterapeuta)

Juliana Sobral Antunes ${ }^{1}$

(Fisioterapeuta)

Lucinéia de Fátima Chasko Ribeiro (Bióloga)

Rose Meire Costa Brancalhão (Bióloga)

Gladson Ricardo Flor Bertolini (Fisioterapeuta)

1. Universidade Estadual do Oeste do Paraná (UNOESTE), Programa de Biociências e Saúde, PR, Brasil.

\section{Correspondência:}

Gladson Ricardo Flor Bertolini Laboratório de Estudo das Lesões e Recursos Fisioterapêuticos da UNIOESTE. Rua Universitária, 2069, Jardim Universitário, Cascavel, PR, Brasil. 85819-110.

gladson_ricardo@yahoo.com.br

\section{RESUMO}

Introdução: A crioterapia é uma modalidade terapêutica que visa reduzir processos álgicos e inflamatórios, sendo que a imersão é considerada a forma mais eficaz; no entanto, a literatura apresenta possíveis efeitos deletérios com relação à aplicação da crioterapia em nervos superficiais. Objetivo: Avaliar o efeito da crioterapia em modelo experimental de compressão do nervo isquiático em ratos Wistar, por meio de análise funcional e morfológica. Métodos: Foram utilizados 42 ratos, sendo seis animais por grupo: G1 - controle, submetido à eutanásia no $15^{\circ}$ dia de pós-operatório (PO); $G 2, G 3$ e G4 - submetidos à compressão do nervo isquiático, submetidos à eutanásia no $3^{\circ}$, $8^{\circ}$ e $15^{\circ}$ dias de $\mathrm{PO}$, respectivamente; G5, G6 e G7 - submetidos à compressão do nervo isquiático e tratados com crioterapia, submetidos à eutanásia no $3^{\circ}, 8^{\circ}$ e $15^{\circ}$ dias de $\mathrm{PO}$, respectivamente. As avaliações do índice funcional do isquiático (IFC) e do teste de incapacidade funcional aconteceram nos momentos pré-lesão, no $2^{\circ}$ de PO e no dia da eutanásia em cada grupo com lesão. Após o período de intervenção, os animais foram devidamente anestesiados e o nervo isquiático distal ao procedimento de compressão foi dissecado e coletado para análise morfológica. A análise estatística foi realizada pelo teste de ANOVA mista, com nível de significância de 5\%. Resultados: Houve diminuição do IFC após a lesão e o teste de incapacidade funcional mostrou aumento do tempo de elevação da pata. Com relação à análise morfológica, o G1 apresentou fibras nervosas com aspecto normal e nos grupos com lesão houve degeneração nervosa, sendo que o G6 teve uma discreta recuperação das fibras nervosas, além de leve regeneração no G4 e G7. Conclusão: A crioterapia não foi eficaz para recuperar os parâmetros funcionais analisados, entretanto, houve discreta melhora dos aspectos morfológicos do grupo submetido à eutanásia no $8^{\circ}$ dia de PO. Nível de Evidência ll; Estudos terapêuticos - Investigação dos resultados do tratamento.

Descritores: Síndromes de compressão nervosa; Nervo isquiático; Modalidades de fisioterapia; Crioterapia; Medição da dor.

\section{ABSTRACT}

Introduction: Cryotherapy is a therapeutic modality that aims to reduce inflammatory and painful processes, with immersion being considered the most effective form; however, the literature has possible deleterious effects related to the application of cryotherapy to superficial nerves. Objective: To evaluate the effect of cryotherapy in experimental model of sciatic nerve compression in Wistar rats, through morphologic and functional analysis. Methods: Forty-two rats were used, six animals per group: G1 - control euthanized on the $15^{\text {th }}$ postoperative day (PO); G2, G3 and G4 - submitted to sciatic nerve compression, euthanized at $3^{\text {rd }}, 8^{\text {th }}$ and $15^{\text {th }}$ PO days, respectively; G5, G6 and G7 - submitted to sciatic nerve compression and treated with cryotherapy, euthanized at $3^{\text {rd }}, 8^{\text {th }}$ and $15^{\text {th }} P O$ days, respectively. The assessments of sciatic functional index (SFI) and the functional disability test took place at the pre-injury, $2^{\text {nd }} P O$ and on the day of euthanasia in each group with injury. After the intervention period, the animals were anesthetized properly and the sciatic nerve distal to the compression procedure was dissected and collected for morphological analysis. Statistical analysis was by the mixed ANOVA test with a significance level of 5\%. Results: There was a decrease of SFI after injury and the functional disability test showed an increase in paw elevation time. Regarding the morphological analysis, the G1 showed normal nerve fibers and in the groups with lesion, there was nerve degeneration, G6 had a slight recovery of the nerve fibers, besides mild regeneration in G4 and G7. Conclusion: Cryotherapy was not effective to recover the functional parameters analyzed; however, there was a slight improvement in the morphological aspects of the group euthanized on the $8^{\text {th }}$ PO day. Level of Evidence II; Therapeutic studies - Investigating the results of treatment.

Keywords: Nerve compression syndromes; Sciatic nerve; Physical therapy modalities; Cryotherapy; Pain measurement.

\section{RESUMEN}

Introducción: La crioterapia es una modalidad terapéutica que busca reducirprocesos de dolor e inflamatorios, siendo que la inmersión es considerada la forma más eficaz; sin embargo, la literatura presenta posibles efectos deletéreos con relación a la aplicación de la crioterapia en nervios superficiales. Objetivo: Evaluar el efecto de la crioterapia en modelo experimental de compresión del nervio ciático en ratas Wistar, por medio de análisis funcional y morfológico. Métodos: Se utilizaron 42 ratas, siendo seis animales por grupo: G1 - control, sometido a la eutanasia en el $15^{\circ}$ día de postoperatorio (PO); G2, G3 y G4 - sometidos a la compresión del nervio ciático, sometidos a la eutanasia en los 30, 80 y $15^{\circ}$ días de PO, respectivamente; G5, G6 y G7 - sometidos a la compresión del nervio ciático y tratados con crioterapia, sometidos 
a la eutanasia en los 30, $8^{\circ}$ y $15^{\circ}$ días de PO, respectivamente. Las evaluaciones del índice funcional del ciático (IFC) y la prueba de incapacidad funcional ocurrieron en los momentos pre-lesión, en el $2^{\circ}$ día de PO y el día de la eutanasia en cada grupo con lesión. Después del período de intervención, los animales fueron debidamente anestesiados y el nervio ciático distal al procedimiento de compresión fue disecado y recogido para análisis morfológico. El análisis estadístico fue realizado por la prueba de ANOVA mixta, con un nivel de significancia del 5\%. Resultados: Hubo disminución del IFC después de la lesión y la prueba de incapacidad funcional mostró aumento del tiempo de elevación de la pata. En cuanto al análisis morfológico, el G1 presentó fibras nerviosas con aspecto normal y en los grupos lesionados hubo degeneración nerviosa, siendo que el G6 tuvo una discreta recuperación de las fibras nerviosas, además de ligera regeneración en el G4 y G7. Conclusión: La crioterapia no fue eficaz para recuperar los parámetros funcionales analizados, sin embargo, hubo discreta mejora de los aspectos morfológicos del grupo sometido a la eutanasia en el 8 o día de PO. Nivel de Evidencia II; Estudios terapéuticos - Investigación de los resultados del tratamento.

Descriptores: Síndromes de compresión nerviosa; Nervio ciático; Modalidades de fisioterapia; Crioterapia; Dimensión del dolor.

\section{INTRODUÇÃO}

As lesões nervosas periféricas variam em extensão e gravidade, as quais podem ocorrer por trauma, compressão e isquemia, levando a déficits neurológicos tanto motores como sensoriais. ${ }^{1}$ Estas lesões afetam a qualidade de vida, incluindo a perda total ou parcial de atividades produtivas, o que origina importantes consequências econômicas e sociais para a população. ${ }^{2}$ Dependendo do tipo da lesão, pode haver recuperação espontânea ou ser necessário uma intervenção cirúrgica ou terapêutica para minimizar os danos causados pela lesão. ${ }^{3-5}$

Em relação ao tratamento, existem diversas técnicas que podem ser utilizadas dentro da fisioterapia, como a crioterapia, um tipo de termoterapia em que se aplica o frio como agente terapêutico. ${ }^{6}$ A crioterapia induz a redução da temperatura corporal, que diminui a taxa metabólica dos tecidos, as reações enzimáticas, a liberação de histamina, leva à vasoconstrição com consequente diminuição do fluxo sanguíneo, permeabilidade da membrana e edema. ${ }^{7}$ Além disso, apresenta efeitos analgésicos, por meio da ação sobre a velocidade de condução nervosa e inibição da transmissão de sinais de dor para o corno posterior da medula espinal. ${ }^{8} \mathrm{O}$ frio também pode agir como um estimulante contra irritante pelas vias centrais da dor, que então ativam as vias descendentes inibitórias, bloqueando a transmissão da dor para o cérebro. ${ }^{9}$

A crioterapia pode ser aplicada por diferentes métodos como compressas frias, massagem com gelo, bolsas e dispositivos de compressão, ${ }^{10}$ e dentre as diversas formas existe a imersão com água e gelo considerada a mais eficaz, pois abrange uma grande área para tratamento de lesões. No entanto a literatura apresenta possíveis efeitos deletérios com relação a aplicação da crioterapia em nervos superficiais., 6,11,12

Desta maneira, devido a carência de estudos a respeito da crioterapia em lesões nervosas periféricas e se sua aplicação apresenta algum efeito sobre o aspecto morfológico e funcional, justifica-se o presente estudo. A partir disso, o objetivo foi avaliar o efeito da crioterapia em modelo experimental de compressão do nervo isquiático em ratos Wistar, por meio de análise funcional e morfológica.

\section{MATERIAIS E MÉTODOS}

O grupo amostral foi composto por 42 ratos da linhagem Wistar, machos, com peso de $327 \pm 24,84 \mathrm{~g}$ e idade de 10 semanas, mantidos no Biotério setorial do Laboratório de Estudo das Lesões e Recursos Fisioterapêuticos (LELRF), em caixas de polipropileno, em ambiente com temperatura de $23 \pm 1^{\circ} \mathrm{C}$ e fotoperíodo de 12 horas, recebendo água e ração ad libitum. O projeto foi aprovado pelo Comitê de Ética em Uso de Animais (CEUA) da Unioeste (15/07/2014).
O critério utilizado para o número de animais foi baseado no princípio dos 3Rs (Reduction, Refinement, Replacement), ou seja, redução, refinamento e substituição, que visa a redução da quantidade de animais, sem prejudicar a comprovação científica dos resultados. ${ }^{13}$

\section{Grupos experimentais}

Os animais foram separados aleatoriamente em sete grupos com seis animais em cada grupo:

- Grupo Controle (C) - Composto por animais que não foram submetidos à compressão nervosa e crioterapia, eutanasiados no $15^{\circ}$ dia após início do experimento;

- Grupo Lesão 3oPO (L3) - submetidos à compressão nervosa, sem nenhuma forma de tratamento, eutanasiados no $3^{\circ}$ pós-operatório (PO);

- Grupo Lesão 80PO (L8) - submetidos à compressão nervosa, sem tratamento, eutanasiados no $8 \circ \mathrm{PO}$;

- Grupo Lesão 15०PO (L15) - submetidos à compressão nervosa, sem tratamento, eutanasiados no $15^{\circ} \mathrm{PO}$;

- Grupo Lesão com crioterapia 3oPO (LCrio3) - submetidos à compressão nervosa e tratamento com crioterapia, eutanasiados no $3^{\circ} \mathrm{PO}$;

- Grupo Lesão com crioterapia 8०PO (LCrio8) - submetidos à compressão nervosa e tratamento com crioterapia, eutanasiados no 80 PO;

- Grupo Lesão com crioterapia 150PO (LCrio15) - submetidos à compressão nervosa e tratamento com crioterapia, eutanasiados no 150 PO.

Prévio ao procedimento cirúrgico, de compressão do nervo isquiático, os animais foram pesados e anestesiados com cloridrato de quetamina (95 mg/Kg) e cloridrato de xilazina (12 mg/Kg) por via intraperitoneal. Após a aplicação da anestesia e a verificação do estado de consciência do animal, os animais foram posicionados em decúbito ventral e foi realizada a tricotomia no local do procedimento cirúrgico.

Em seguida foi realizada uma incisão paralela às fibras do músculo bíceps femoral, expondo o nervo isquiático do membro pélvico direito, com subsequente compressão do mesmo, com o auxílio de pinça hemostática, por um período de 30 segundos. A pressão de pinçamento foi padronizada, utilizando-se como referência o fechamento do segundo dente da cremalheira e realizado pelo mesmo pesquisador. ${ }^{14}$ Após a compressão do nervo, foi realizada sutura epineural, com fio de Nylon 10.0, para marcar a região lesionada. ${ }^{15}$ Por fim, foi realizada a sutura externa com fio catgut 4.0, aplicado iodo sobre a incisão e os animais foram alojados nas mesmas condições pré-cirúrgicas, separados de acordo com o seu grupo experimental.

Para a realização do tratamento, os animais foram contidos manualmente e o membro pélvico direito imerso em recipiente de $1.440 \mathrm{~cm}^{3}$ $(20 \mathrm{~cm} \times 12 \mathrm{~cm} \times 6 \mathrm{~cm})$, contendo água e gelo, durante 20 minutos, em temperatura de $5 \pm 2{ }^{\circ} \mathrm{C}$ verificada pelo termômetro Incoterm ${ }^{\circledR}{ }^{16}$ Em 
G5, G6 e G7 o tratamento ocorreu logo após término do procedimento cirúrgico. Assim, em G5 foi realizada uma única sessão de tratamento e, para G6 e G7, o tratamento continuou do $3^{\circ}$ dia de PO e terminou no 70 dia PO. Os animais de G2, G3 e G4 também foram posicionados de acordo com o protocolo de tratamento, embora o recipiente não contivesse água ou gelo.

Para avaliar a funcionalidade do nervo, foi utilizado o Índice funcional do isquiático, pelo qual foram obtidas imagens da marcha do animal por uma câmera acoplada a uma esteira com armazenamento de imagens em um computador. O método foi baseado na medida de parâmetros pré-estabelecidos nas impressões da pata pélvica dos animais, do lado normal e do lado experimental, sendo medido: o comprimento da pata (PL ou print lenght) que é a distância entre os dois pontos mais extremos da pegada (extremidade do 30 dedo ao calcâneo); o espalhar total dos dedos (TS ou total spread) que é a distância entre $1^{\circ}$ ao $5^{\circ}$ dedo; e o espalhar dos dois dedos intermediários (IT ou intermediate toes) que é a distância entre o $2^{\circ}$ e $4^{\circ}$ dedos. ${ }^{17,18}$

Tais medidas foram realizadas pelo programa Image-Pro Plus 6.0 (MediaCybernetics, Inc. ${ }^{\circledR}$ - Estados Unidos da América) e analisadas no programa Microsoft Office Excel 2007 com a seguinte fórmula: ${ }^{19}$

$I F C=-38,8 \cdot \frac{E P L-N P L}{N P L}+109,5 \cdot \frac{E T S-N T S}{N T S}+13,3 \cdot \frac{E I T-N I T}{N I T}-8,8$

Os resultados foram expressos em percentual negativo da função normal, em que 0 (zero) corresponde a função normal e -100 (menos cem) à disfunção total. ${ }^{18}$

Esta avaliação foi realizada nos seguintes momentos: pré-lesão, no $2^{\circ}$ dia após a lesão (2०PO) e no dia correspondente à eutanásia de cada grupo lesionado $\left(3^{\circ}, 8^{\circ}\right.$ e 15ㅇ), sendo que o G1 (controle) foi avaliado nos mesmos momentos que os grupos submetidos à lesão, porém com eutanásia no 150PO.

A avaliação da nocicepção foi realizada por meio do teste de incapacidade funcional que se caracteriza por um cilindro metálico em movimento e um programa de computador, com conexão a uma bota metálica adaptada à pata do animal. ${ }^{20}$ Os animais deambularam sobre o cilindro, de aproximadamente $30 \mathrm{~cm}$ de diâmetro recoberto por tela de aço inoxidável, que por meio de um motor elétrico realizava três rotações por minuto. Nos membros pélvicos dos animais foram adaptadas botas, confeccionadas com metal, que conduziam a informação da pata direita, por meio de um fio a um computador, o qual apresentava um programa que mostra os valores de tempo de elevação da pata (TEP) do animal deambulando sobre o cilindro em um minuto; o membro pélvico esquerdo também foi mantido conectado a uma bota, porém sem entrada de informações para o computador. Esta avaliação foi realizada posteriormente ao IFC, sempre nos mesmos dias.
Após o período de intervenção, os animais foram devidamente anestesiados e foi coletado um fragmento de 0,5 centímetro $(\mathrm{cm})$ do nervo isquiático direito de cada animal, distal ao procedimento de compressão, demarcada pela sutura epineural. Posteriormente, ainda sob o efeito da anestesia, os animais foram decapitados em guilhotina.

Para a análise histológica, o fragmento do nervo isquiático foi fixado em Bouin, desidratado, diafanizado, infiltrado e emblocado em parafina, sendo seccionado transversalmente em 5 um de espessura em micrótomo Olympus CUT 4055. Posteriormente, foi realizado o procedimento para confecção das lâminas histológicas, com coloração em hematoxilina e eosina (HE), nas quais foram observado e descrito às seguintes estruturas: tecido conjuntivo (epineuro, perineuro e endoneuro), fibra nervosa, infiltrado inflamatório e células de Schwann, sendo fotomicrografadas no aparelho Olympus BX 50. Além de confecção de lâminas com coloração em Tricrômico de Masson, as quais também foram fotomicrografadas, com objetiva de 100x, para análise da porcentagem do tecido conjuntivo endoneuro, em que se selecionava o tecido conjuntivo por meio do programa GIMP 2.8 e o número de pixels obtido foi dividido pelo número total de pixels da imagem e após multiplicado por 100.

\section{Análise estatística}

Os resultados são apresentados por meio de gráficos de linha, sendo analisados com auxílio do programa SPSS 18.0 (Statistical Package for the Social Sciences). A comparação entre os grupos e avaliações, foi realizada entre o grupo controle, lesão e lesão com crioterapia, eutanasiados no mesmo PO. Para a normalidade dos dados foi utilizado o teste Shapiro-wilk, como houve normalidade foi aplicado o teste estatístico ANOVA modelo misto, com pós teste de Bonferroni e para análise do tecido conjuntivo foi utilizado ANOVA unidirecional com pós teste Tukey, sendo o nível de significância aceito de $a=0,05$.

\section{RESULTADOS}

De acordo com a análise do IFC para C, L3 e LCrio3 houve diferença significativa $[F(2 ; 30)=18 ; p<0,001]$, em que L3 e LCrio3 apresentaram valores menores do IFC comparado com $C(p<0,001)$ e as avaliações realizadas no 2०PO e no dia da eutanásia (3०PO) também mostraram valores menores que a avaliação pré-lesão ( $p<0,0001)$ (Figura 1A).

Ao analisar C, L8 e LCrio8, também apresentaram diferença significativa $[F(2 ; 30)=72,3 ; p<0,001]$. Os valores do IFC de L8 e LCrio8 foram menores comparados ao $C(p<0,0001)$. As avaliações do $2^{\circ}$ e $8 \circ \mathrm{PO}$ mostraram IFC menor comparado com a pré-lesão ( $p<0,0001)$, além da avaliação do $8 \circ \mathrm{PO}$ que obteve valor menor que a do 2०PO ( $\mathrm{p}=0,037)$ (Figura 1B).

Os dados do C, L15 e LCrio15 mostraram diferença estatística $[F(2 ; 30)=29 ; p<0,001]$. L15 e LCrio15 obtiveram IFC menor que $C(p<0,001)$ e as avaliações do $2^{\circ}$ e $15^{\circ} \mathrm{PO}$ apresentaram valores menores que avaliação pré-lesão ( $p<0,0001)$. (Figura 1C)

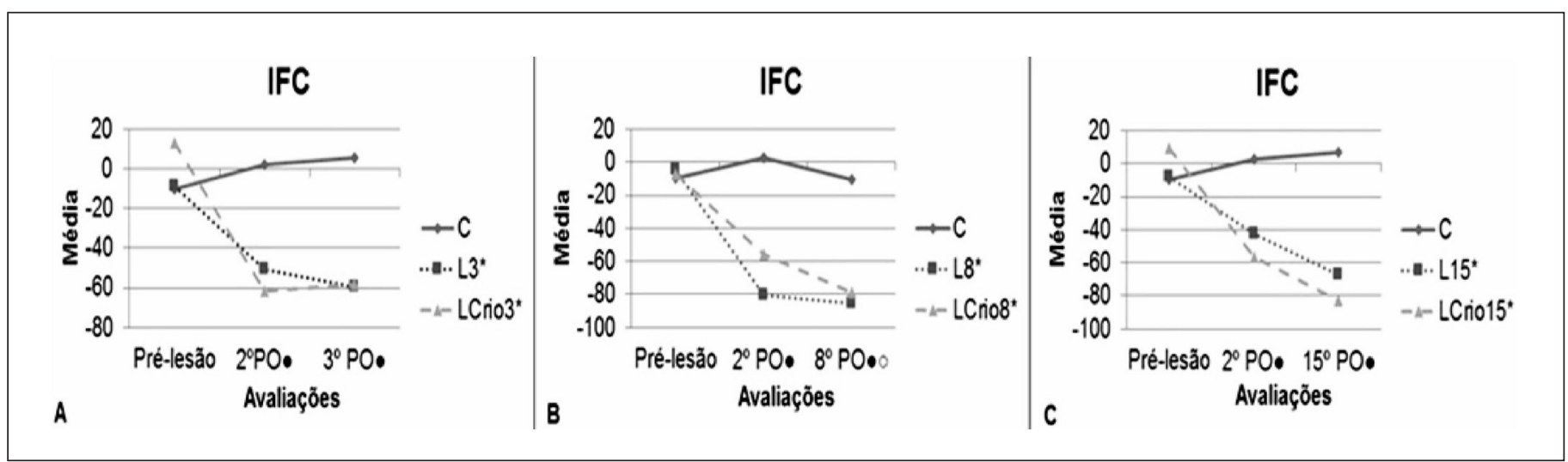

Figura 1. A, B e C - (*) diferença significativa comparada com C, $(\bullet)$ diferença significativa comparada com avaliação pré-lesão e (o) diferença significativa comparada com avaliação no $2 \circ \mathrm{PO}$. 
Com relação ao C, L3 e LCrio3, os resultados demonstraram diferença significativa $[F(2 ; 30)=19,1 ; p<0,001]$, na avaliação do $2 \circ \mathrm{PO}$ e do dia da eutanásia (3०PO) em que o TEP foi maior comparado com a pré-lesão $(p<0,001)$ (Figura 2A).

Para C, L15 e LCrio15, observou-se diferença estatística $[F(2 ; 30)=26,9 ; p<0,001]$ na avaliação do $2^{\circ}$ e $8^{\circ} \mathrm{PO}$ que apresentou TEP maior que a pré-lesão $(p<0,0001)$ (Figura 2B).

Ao comparar C, L15 e LCrio15, apresentaram diferença $[F(2 ; 30)=42,7 ; p<0,001]$, na avaliação do $2^{\circ}$ e $15 \circ \mathrm{PO}$ que mostraram valores maiores de TEP comparados com a pré-lesão $(p<0,0001)$, além da avaliação do 15०PO, que obteve TEP maior que a avaliação do $2 \circ \mathrm{PO}$ $(p=0,005)$. (Figura 2C)

As análises morfológicas do nervo isquiático do C (controle) revelaram o aspecto morfológico normal, com seus envoltórios conjuntivos íntegros, epineuro, perineuro e endoneuro (Figura 3A). As fibras nervosas também se mostravam normais, com os axônios envoltos por bainha de mielina, e núcleos de células de Schwann na periferia da fibra (Figura 3B).

Em todos os grupos submetidos à lesão, verificou-se que o modelo de compressão nervosa por esmagamento utilizado neste estudo, foi eficiente para causar degeneração nervosa tipo axoniotmese (Figura $3 C$ a H), pois os nervos isquiáticos exibiram eventos morfológicos característicos da degeneração Walleriana.

No grupo lesão (L3) bem como no grupo lesão e submetido à crioterapia (LCrio3), eutanasiados no $3^{\circ}$ PO, foram evidentes áreas com degeneração nervosa, e inicio da resposta inflamatória, com a presença de macrófagos e células gigantes multinucleadas (Figura 3C e D), não havendo diferenças morfológicas relevantes no grupo tratado em relação ao lesão.

Com relação aos grupos lesão (L8) e crioterapia (LCrio8), eutanasiados no $8^{\circ}$ dia após a lesão, foram encontrados aspectos morfológicos semelhantes ao L3 e LCrio3, entretanto, em L8 houve um maior acometimento do nervo com aumento de fibras degeneradas e infiltrado inflamatório (Figura 3E). Já no LCrio8, foi verificada uma leve diminuição do infiltrado inflamatório, assim como uma discreta recuperação das fibras nervosas. (Figura 3F)

Já em L15 e LCrio15, eutanasiados no $15^{\circ} \mathrm{PO}$, foram observados aspectos morfológicos semelhantes, com fibras nervosas ainda degeneradas, mas um grande número em regeneração e uma diminuição no número de células inflamatórias, quando comparado com os demais grupos lesionados e tratados. No entanto não houve diferenças morfológicas significativas entre o grupo lesão (L15) e grupo lesionado e tratado com crioterapia (LCrio15). (Figura 3G e H)
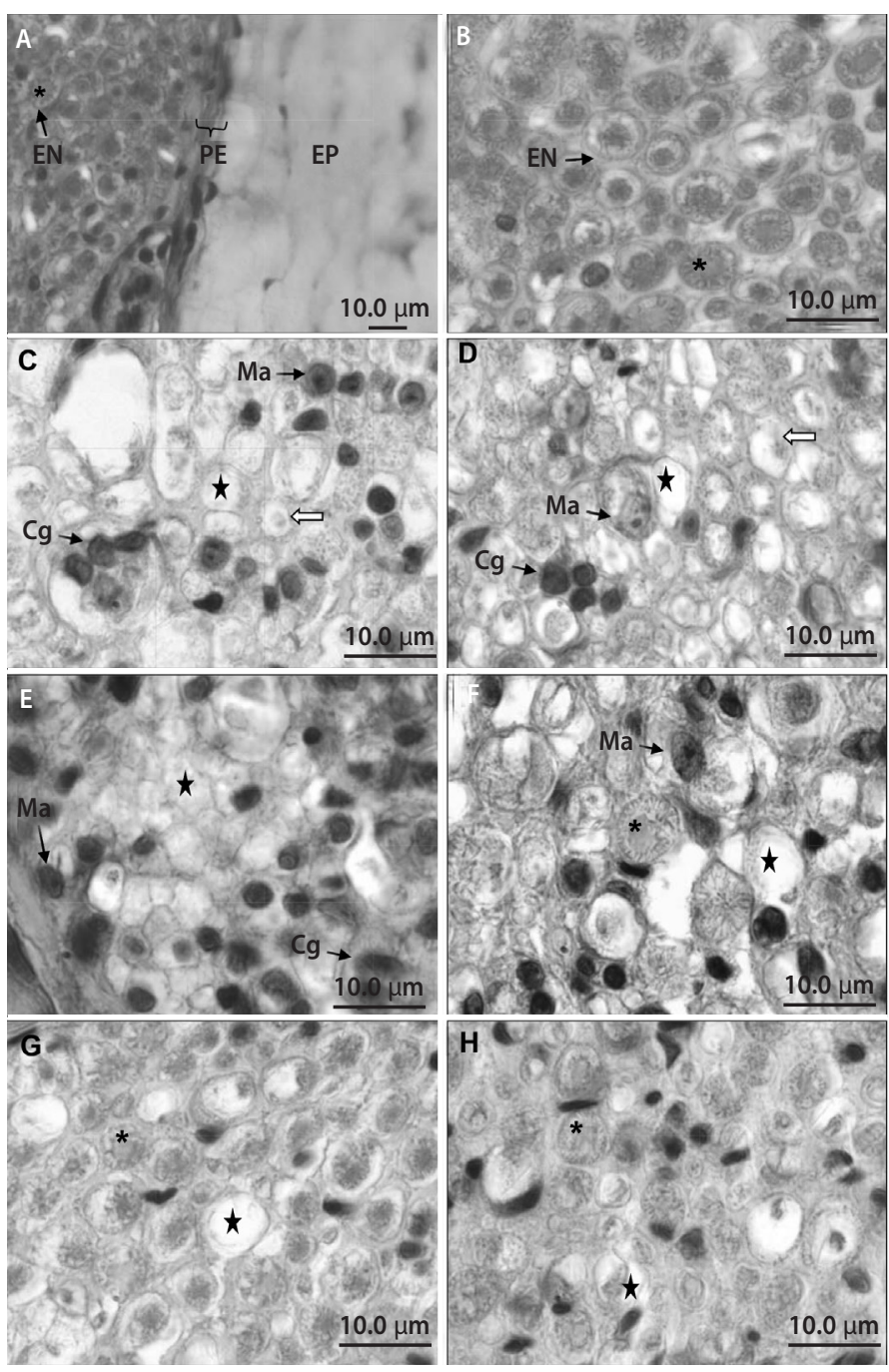

Figura 3. A a H. Fotomicrografias de corte transversal do segmento distal do nervo isquiático de ratos Wistar, coloração hematoxilina e eosina (HE). Em A e B grupo controle (C) no qual se observa bainhas conjuntivas: epineuro (EP), perineuro (PE) e endoneuro (EN), fibra nervosa $\left(^{*}\right)$ e núcleos de possíveis células de Schwann $(>)$. Em C e D, grupo L3 e LCrio3 (lesão e lesão com crioterapia respectivamente, eutanasiados no $3^{\circ}$ dia pós-operatório), nota-se a presença de uma hipercelularidade com macrófagos (Ma), células gigantes multinucleadas (Cg), fibras degeneradas (estrela) e em degeneração (seta branca). Em E, grupo L8 (lesão e eutanasiado no $8^{\circ}$ dia pós-operatório), mostra um grande número de células inflamatórias (Ma e Cg) e fibras degeneradas (estrela). Em F, grupo LCrio8 (lesão e tratado com crioterapia, eutanasiado no 8dia pós-operatório), mostrando uma leve melhora, com diminuição das células inflamatórias e regeneração das fibras nervosas. Nas figuras G (L15) e H (LCrio 15), lesão e lesão tratado com crioterapia respectivamente, eutanasiados no 150PO, apresentam características morfológicas semelhantes, ainda com algumas fibras degeneradas (estrela), diminuição de células inflamatórias, fibras nervosas normais (*) e núcleos de célula de Schwann (

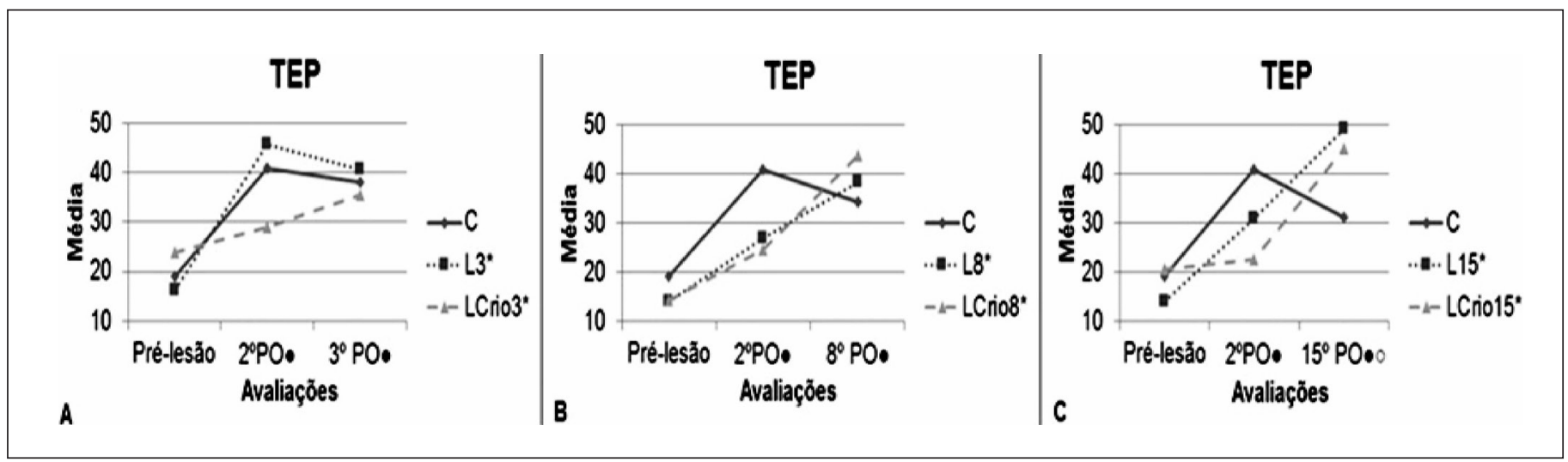

Figura 2. A, B e C - (•) diferença significativa comparada com avaliação pré-lesão e (o) diferença comparada com avaliação no 2oPO. 
Com relação ao tecido conjuntivo, foi realizada uma análise quantitativa para complementar os dados morfológicos, na qual não houve diferença significativa entre os grupos analisados $(F=0,59 ; p>0,5 ; F=1,7 ; p>0,2$ e $F=0,15 ; p>0,8$, correspondendo respectivamente às comparações dos grupos do $30,8^{\circ}$ e $\left.15^{\circ} \mathrm{PO}\right)$.

\section{DISCUSSÃO}

A lesão do nervo periférico pode ser classificada em três tipos: neuropraxia, axoniotmese e neurotmese. Neste estudo utilizou-se a lesão por compressão, a qual provoca axoniotmese, em que a bainha de mielina e os axônios são lesionados, sendo que ocorre a degeneração do axônio no segmento distal a lesão, denominada degeneração Walleriana, ${ }^{21,22}$ e como possível tratamento foi utilizado a crioterapia que é recomendada após lesão, para minimizar os efeitos negativos do processo inflamatório. ${ }^{9}$

A partir do modelo de lesão proposto, houve redução da função avaliada pelo IFC que é um método quantitativo, confiável e reprodutível para avaliar o grau da lesão e de recuperação. ${ }^{17}$ Entretanto, independente do tratamento com crioterapia, não houve recuperação funcional, o que pode ter acontecido devido às alterações morfológicas de cada período analisado (3०, $8^{\circ}$ e $\left.15^{\circ} \mathrm{PO}\right)$, como a presença de fibras nervosas degeneradas e infiltrado inflamatório, denotando uma relação entre as alterações morfológicas do nervo com sua função.

Outro teste realizado foi o de incapacidade funcional, que verificou o tempo de elevação da pata, o qual aumentou, de acordo com as avaliações realizadas pós-lesão, ou seja, os animais apresentaram aumento da nocicepção, independente do tratamento, o que pode ter ocorrido devido ao acúmulo de substâncias álgicas produzidas durante o processo inflamatório, como a bradicinina ${ }^{19}$ sendo que no presente estudo foram observadas características morfológicas inflamatórias que se mantiveram até o $15^{\circ} \mathrm{PO}$, as quais serão discutidas posteriormente.

Esses resultados são semelhantes ao estudo de Bertolini et al., ${ }^{23}$ em que ratos foram submetidos à compressão do nervo isquiático com protocolo de amarria, entretanto, tratados com corrente de alta voltagem e avaliados também pelo teste de incapacidade funcional, o qual verificou que não houve diminuição da nocicepção. Sendo que isso pode ter auxiliado na diminuição do índice funcional do isquiático do presente estudo, pois devido ao quadro nociceptivo, os animais podem apresentar alteração na marcha, prejudicando a funcionalidade, sem descartar a alteração motora produzida pela compressão nervosa.

De acordo com a morfologia, observou-se que o modelo de lesão levou a degeneração Walleriana, apresentando hipercelularidade, incluindo a proliferação de células de Schwann, de macrófagos e de células gigantes multinucleadas para região distal à lesão. Estas células possuem a função de fagocitar os restos mielínicos, além disso, as células de Schwann secretam fatores de crescimento para promover a regeneração das fibras nervosas. ${ }^{21}$

Os grupos L3 e LCrio3, apresentaram fibras degeneradas, em degeneração e células do processo inflamatório, isso acontece porque a degeneração se dá logo após a lesão nervosa e o recrutamento de macrófagos e células de Schwann acontece após 48h da lesão, ${ }^{21}$ sendo que somente um dia de crioterapia (LCrio3), não foi suficiente para alterar o aspecto morfológico do nervo neste período.

O grupo lesão eutanasiado no 8०PO (L8) mostrou um aumento do processo inflamatório com fibras degeneradas, pois neste período aumenta o recrutamento celular para eliminar os restos mielínicos, ${ }^{22}$ entretanto, o grupo tratado com crioterapia (LCrio8), apresentou diminuição do processo inflamatório e discreta regeneração das fibras nervosas, isso pode ter acontecido devido o tratamento que promove a vasoconstrição, que diminui a permeabilidade celular e o processo inflamatório, o que consequentemente pode ter auxiliado no começo da regeneração. ${ }^{11}$

Os animais de L15 e LCrio15, apresentaram fibras degeneradas, regeneradas e diminuição das células inflamatórias. Segundo Sta et al., ${ }^{23}$ aproximadamente duas semanas após a lesão tem início a regeneração nervosa, embora ainda estejam presentes os restos mielínicos e processo inflamatório. No presente estudo o grupo tratado com crioterapia (LCrio15) não se mostrou diferente do grupo lesão (L15), provavelmente pelo tempo de tratamento, visto que a crioterapia quando aplicada durante seis dias foi eficiente na diminuição da intensidade da lesão, como verificado no grupo LCrio8, porém não foi eficaz na manutenção dos efeitos quando interrompida na fase mais tardia, como aconteceu nos animais de LCrio15. Assim, sugere-se que a crioterapia seria interessante como medida terapêutica na primeira semana e, após isso, a aplicação de técnicas adicionais que possam acelerar a regeneração, ou ainda que houvesse continuidade do tratamento até o $15^{\circ}$ dia após a lesão.

Complementando os dados morfológicos foi realizada a porcentagem de tecido conjuntivo endoneural, a qual não mostrou diferença significativa entre os grupos, ou seja, a lesão afetou apenas a morfologia da fibra nervosa e não o seu endoneuro.

Contudo, salienta-se como limitação do presente estudo, a ausência de análises morfométricas e imuno-histoquímicas, sendo essas sugestões para próximos estudos, as quais podem complementar os resultados e compreender melhor o efeito da crioterapia na lesão nervosa periférica.

\section{CONCLUSÃO}

Conclui-se que a crioterapia, durante 20 minutos em imersão do membro pélvico, não foi eficaz para recuperar os parâmetros funcionais analisados (índice funcional do isquiático bem como teste de incapacidade funcional), entretanto, houve melhora dos aspectos morfológicos do grupo eutanasiado no 80 pós-operatório, com diminuição do infiltrado inflamatório e discreta recuperação das fibras nervosas.

\section{AGRADECIMENTOS}

À Coordenação de Aperfeiçoamento de Pessoal de Nível Superior (CAPES), pela bolsa de mestrado e Fundação Araucária de Apoio ao Desenvolvimento Científico e Tecnológico, pelos recursos financeiros do projeto.

Todos os autores declararam não haver qualquer potencial conflito de interesses referente a este artigo.

CONTRIBUIÇÕES DOS AUTORES: Cada autor contribuiu individual e significativamente para o desenvolvimento de manuscrito. JK (0000-0002-3714-0002)*: foi a principal contribuinte na redação do manuscrito; JK, LV (0000-0003-3881-896X)*, CMMK (0000-0002-1586-4720)* e JSA (0000-0002-7848-9023)*: realizaram os procedimentos de lesão experimental, tratamento, coletas e análises de laminário, e aprovação da versão final do manuscrito; LFCR (0000-0001-5174-7399)* e RMCB (0000-0002-5344-5076)*: realizaram avaliação crítica do laminário, com participação ativa na discussão dos resultados e aprovação da versão final do manuscrito. GRFB (0000-0003-0565-2019)*: participou da concepção da pesquisa, análise estatística e interpretação de dados, além de revisão da versão final. *ORCID (Open Researcher and Contributor ID). 


\section{REFERÊNCIAS}

1. Brull R, Hadzic A, Reina MA, Barrington, MJ. Pathophysiology and etiology of nerve injury following peripheral nerve blockade. Reg Anesth Pain Med. 2015;40(5):479-90.

2. Sebben $A D$, Lichtenfels M, Silva JL. Regeneração de nervos periféricos: terapia celular e fatores neurotróficos. Rev Bras Ortop. 2011;46:643-9.

3. Martins RS, Bastos D, Siqueira MG, Heise CO, Teixeira MJ. Traumatic injuries of peripheral nerves: a review with emphasis on surgical indication. Arq Neuropsiquiatr. 2013;71(10):811-4.

4. Valls-Sole J, Castillo CD, Casanova-Molla J, Costa J. Clinical consequences of reinnervation disorders after focal peripheral nerve lesions. Clin Neurophysiol. 2011;122(2):219-28.

5. Ribak S, Silva Filho PR, Tietzmann A, Hirata HH, Mattos CA, Gama SA. Use of superficial peroneal nerve graft for treating peripheral nerve injuries. Rev Bras Ortop. 2016;51(1):63-9.

6. Gutiérrez Espinoza HJ, Lavado Bustamante IP, Mendéz Pérez SJ. Revisión sistemática sobre el efecto analgésico de la crioterapia en el manejo del dolor de origen músculo esquelético. Rev Soc Esp Dolor. 2010;17(5):242-52.

7. Matos F, Neves EB, Norte M, Rosa C, Reis VM, Vet al. The use of thermal imaging to monitoring skin temperature during cryotherapy: a systematic review. Infrared Phys Technol. 2015;73:194-203.

8. Costello JT, McInerney CD, Bleakley CM, Selfe J, Donnelly AE. The use of thermal imaging in assessing skin temperature following cryotherapy: a review. J Therm Biol. 2012;37:103-10.

9. Corti L. Nonpharmaceutical approaches to pain management. Top Companion Anim Med. 2014;29:24-8.

10. Martimbianco AL, Gomes da Silva BN, Carvalho AP de, Silva D, Torloni MR, Peccin MS. Effectiveness and safety of cryotherapy after arthroscopic anterior cruciate ligament reconstruction: a systematic review of the literature. Phys Ther Sport. 2014;15(4):261-8.

11. Hanks J, Levine D, Bockstahler B. Physical agent modalities in physical therapy and rehabilitation of small animals. Vet Clin North Am Small Anim Pract. 2015;45:29-44.

12. Herrera E, Sandoval MC, Camargo DM, Salvini TF. Effect of walking and resting after three cryotherapy modalities on the recovery of sensory and motor nerve conduction velocity in healthy subjects. Rev Bras Fisioter. 2011;15(3):233-40.
13. Damy SB, Camargo RS, Chammas R, Figueiredo LF. Aspectos fundamentais da experimentação animal em cirurgia experimental. Rev Assoc Med Bras 2010;56(1):103-11.

14. Bridge PM, Ball DJ, Mackinnon SE, Nakao Y, Brandt K, Hunter DA, et al. Nerve crush injuries: a model for axonotmese. Exp Neurol. 1994;127:284-90.

15. Savastano LE, Laurito SR, Fitt MR, Rasmussen JA, Gonzalez Polo V, Patterson SI. Sciatic nerve injury: a simple and subtle model for investigating many aspects of nervous system damage and recovery. J Neurosci Methods. 2014;227:166-80.

16. Moreira NB, Artifon EL, Meireles A, Silva LI, Rosa CT, Bertolini, GR. A influência da crioterapia na dor e edema induzidos por sinovite experimental. Fisioter e Pesqui. 2011;18(1):79-83.

17. De Medinaceli L, Freed WJ, Wyatt RJ. An index of the functional based on measurements condition of rat sciatic nerve made from walking tracks. Exp Neurol. 1982;77:634-43.

18. Monte-Raso V V, Fonseca MC, Mazzer N, Barbieri G, Rosa RC, Zamarioli A, et al. Esteira com velocidade controlada para captação da marcha. Acta Ortop Bras. 2010;18(1):49-53.

19. Bain JR, Mackinnon SE, Hunter DA. Functional evaluation of complete sciatic, peroneal, and posterior tibial nerve lesions in the rat. Plast Reconstr Surg. 1989;83(1):129-38.

20. Tonussi CR, Ferreira SH. Rat knee-joint carrageenin incapacitation test: an objective screen for central and peripheral analgesics. Pain. 1992;48(3):421-7.

21. Wood MD, Kemp SW, Weber C, Borschel GH, Gordon T. Outcome measures of peripheral nerve regeneration. Ann Anat. 2011;193(4):321-33.

22. Wong KM, Babetto E, Beirowski B. Axon degeneration: make the Schwann cell great again. Neural Regen Res. 2017;12(4):518-24.

23. Sta M, Cappaert NL, Ramekers D, Bass F, Wadman WJ. The functional and morphological characteristics of sciatic nerve degeneration and regeneration after crush injury in rats. J Neurosci Methods. 2014;222:189-98. 